\title{
Herausforderungen bei der Kodierung von Paratext am Beispiel Neuer Musik mit Live-Elektronik
}

Daniel Fütterer, Karlsruhe

DOI: $10.25366 / 2020.103$

Zitation: Daniel Fütterer, „Herausforderungen bei der Kodierung von Paratext am Beispiel Neuer Musik mit LiveElektronik", in: Brückenschläge zwischen Musikwissenschaft und Informatik. Theoretische und praktische Aspekte der Kooperation, in Verbindung mit der Fachgruppe Digitale Musikwissenschaft hrsg. von Stefanie Acquavella-Rauch, Andreas Münzmay und Joachim Veit (= Musikwissenschaft: Aktuelle Perspektiven. Bericht über die Jahrestagung der Gesellschaft für Musikforschung 2019 in Paderborn und Detmold, Bd. 3), Detmold, Musikwissenschaftliches Seminar der Universität Paderborn und der Hochschule für Musik Detmold, 2020, S. 141-148, DOI: 10.25366/2020.103

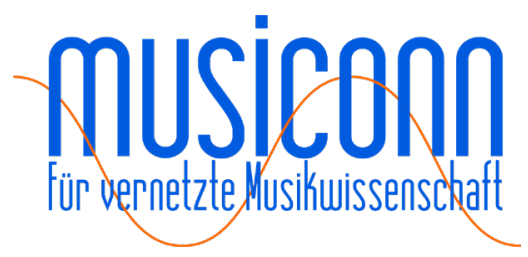




\title{
Herausforderungen bei der Kodierung von Paratext am Beispiel Neuer Musik mit Live-Elektronik
}

\author{
DANIEL FÜTTERER, KARLSRUHE
}

Das laufende Dissertationsprojekt, aus dem das Poster einen Teilaspekt thematisiert, behandelt die Kodierung von Musik, die sich dem gängigen Standard MEI sperrt: ${ }^{1}$ Neue Musik mit LiveElektronik, also (Kunst-)Musik, die mit elektrischen bzw. elektronischen Klangerzeugern und Effektgeräten aufgeführt wird. Um diese Musik für bestimmte Zwecke kodieren zu können, müssen ihre von Normen und Konventionen oft im hohen Maß abweichenden Eigenschaften in Notation und Quellentypen besonders berücksichtigt werden. Im Folgenden soll auf die Bedeutung und Herausforderung der Kodierung von Paratext eingegangen werden.

Neue Musik verweigert sich häufig geradezu, von Standards wie MEI erfasst zu werden. Komponisten brechen bewusst mit der Tradition und Konvention, die als Einschränkung oder überholt gilt und entwerfen eigene Notationsformen, Instrumente und Effektgeräte. 1964 wurden im Kongress "Notation Neuer Musik" im Rahmen der Internationalen Ferienkurse für Neue Musik in Darmstadt die Probleme im Umgang mit der neuartigen, oft grafischen Notation solcher Werke diskutiert. ${ }^{2}$ Das über Jahrhunderte angesammelte Wissen, wie Musik aus Noten ungefähr aufzuführen ist oder über Spieltechniken traditioneller Instrumente, spielt nun keine Rolle mehr. Viele Werke der Neuen Musik verwenden sogar eigens entwickelte Instrumente, Effekte und andere Geräte, deren Bedienung so vielseitig ist, dass reiner Notentext nicht mehr genügt, das nötige Wissen für eine adäquate Aufführung zu vermitteln. ${ }^{3}$

Wo implizites Wissen nicht genügt, werden Informationen explizit oft über Paratext bereitgestellt. Der Begriff Paratext steht hier für Texte bzw. Dokumente, die Bezug zum Werk haben, aber nicht direkter Teil des Notentextes sind. Sie werden unterteilt in Epitext und Peritext. Letzteres sind Texte, die mit dem Basistext noch direkt verbunden sind und die für eine Aufführung mitunter essentiell sind. Als Epitext werden Quellen bezeichnet, die nicht mehr unmittelbar mit

1 MEI bietet Schemata für Neumen, Mensuralnotation und die sog. "Common Western Music Notation" (umgangssprachlich "klassische Musik"). Die Besonderheiten von Musik, die später entstanden ist, sind bisher kein integraler Bestandteil der Schemata. Die Bernd Alois Zimmerman-Gesamtausgabe ist das erste Projekt, das sich mit solcher Musik und MEI auseinandersetzt <http://www.adwmainz.de/projekte/bernd-alois-zimmermann-gesamtausgabe-historisch-kritische-ausgabe-seiner-werke-schriften-und-briefe/information.html> (25.06.2020).

2 Ernst Thomas (Hrsg.), Notation Neuer Musik (= Darmstädter Beiträge zur Neuen Musik IX), Mainz 1965.

3 Bekannte neu entwickelte elektrische Instrumente sind zum Beispiel das Theremin und die verwandten Ondes Martenot; frühe Effektgeräte sind Ringmodulatoren und das Halaphon, welche von Hans Peter Haller im Experimentalstudio des SWR erfunden wurden; vgl. auch Helga de La Motte-Haber, "Neue Musik als mediale Kunst", in: Handbuch Musik und Medien, hrsg. von Holger Schramm, Konstanz 2009, S. 471-494. 
dem Werk zusammenhängen: Interviews, Briefe oder anderes. ${ }^{4}$ In Verbindung mit Live-Elektronik können wichtige Peritexte sein: das Vorwort einer Partitur oder technische Dokumente wie der Aufbauplan einer Bühne mit Position der Musiker und Lautsprecher, eine Liste der verwendeten Hardware, Schaltpläne, Ablaufpläne usw.

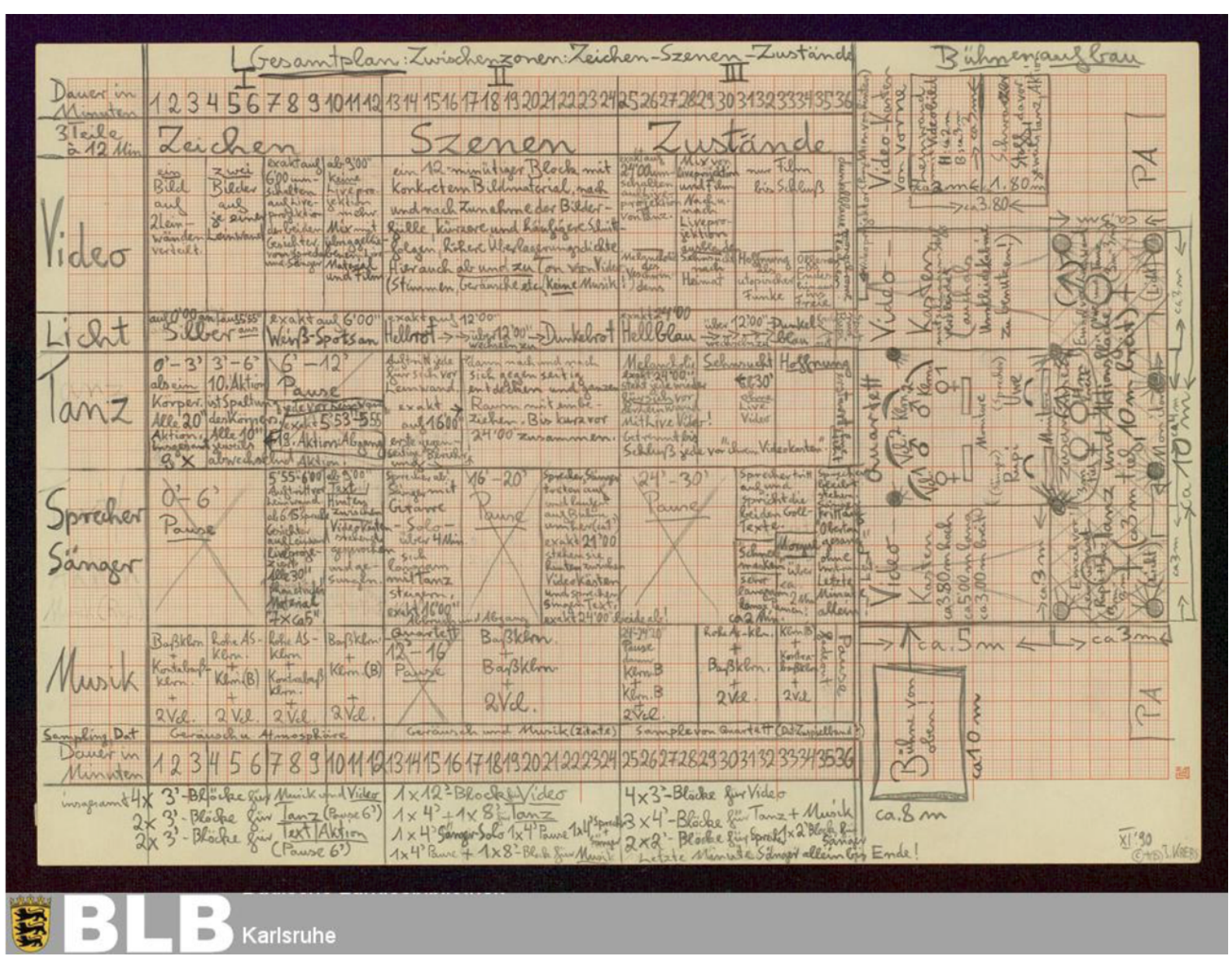

Abbildung1: Quelle: Badische Landesbibliothek Karlsruhe, Signatur K 3353 A 43

In dem auf dem Poster abgebildeten Gesamtplan beschreibt der Komponist Joachim Krebs, dessen Komposition Zwischenzonen als Beispiel dient, sehr anschaulich wichtige Details, die den Notentext des Stückes überlasten könnten, wären sie in ihn integriert, die aber als Vorwort oder Anhang wichtige Informationen liefern und somit in eine Edition einfließen sollten. Sein Werk zeigt sich hier als multimediale Kunst. Während das in einer analogen Edition verhältnismäßig einfach umzusetzen ist, stellt dieser Wunsch in einer genuin digitalen Edition eine Herausforderung dar - je nach ihrer Zielsetzung.

4 Vgl. Gérard Genette, Paratexte. Das Buch vom Beiwerk des Buches (= Suhrkamp-Taschenbuch Wissenschaft 1510), Frankfurt 2001, S. 9-13. 
Digitalisierung bzw. Musikkodierung kann verschiedene Ziele verfolgen, aus denen jeweils eigene Anforderungen entstehen. ${ }^{5}$ Die wohl grundlegendsten Ziele sind der Schutz, die digitale Archivierung und Bereitstellung der Quellen, sei es über Gedächtnisinstitutionen oder Portale von wissenschaftlichen Projekten. Diesen Zielen gerecht zu werden, ist verhältnismäßig unkompliziert, denn, vereinfacht gesagt, genügt ein hochwertiger Scan. Damit ist eine Quelle digitalisiert und kann entsprechend zur Verfügung gestellt werden. Die Verwendbarkeit ist jedoch sehr eingeschränkt, da Bilddateien zunächst keinerlei musikalische Informationen enthalten. Diese müssen erst über Verfahren zur optischen Erkennung erschlossen werden. Digitalisate dienen jedoch als Grundlage für weitere Forschungsarbeiten, die dadurch erleichtert, aufgewertet oder sogar erst ermöglicht werden.

Für eine digitale Edition eines Werkes, das sozusagen "nächstanspruchsvollere" Ziel, muss neben der optischen Erscheinung auch der Inhalt erfasst werden 6 . Im Idealfall werden dazu offene Standards verwendet. Die auch außerhalb der Wissenschaft bekanntesten dürften MIDI und MusicXML sein. Beide sind für ihre grundverschiedenen Einsatzzwecke optimiert und dafür sehr gut geeignet: MIDI für das Übertragen und Protokollieren von Steuersignalen, MusicXML als offenes Austauschformat zwischen ansonsten proprietären Notensatzprogrammen. Für das wissenschaftliche Erfassen einer Quelle mit ihren Metadaten, Querverweisen innerhalb einer Edition, Erfassen und Ergänzen kritischer Informationen und Anmerkungen oder zur Rekonstruktion der Werkgenese, sind beide Formate allerdings ungenügend: Weder MIDI noch MusicXML erlauben es, wissenschaftliche Metadaten etc. anzulegen und enthalten auch längst nicht alle relevanten musikalischen Parameter.

Für solche wissenschaftlichen Ansprüche wurde das XML-Schema MEI konzipiert. Benannt nach der Music Encoding Initiative und inspiriert von TEI, dem Äquivalent zur Kodierung von Textdateien, wird MEI seit 1999 stetig weiterentwickelt und liegt seit 2018 in der Hauptversion 4 vor. ${ }^{7}$ Damit ist es nun möglich, kritische Anmerkungen wie Lesarten, Korrekturen, unklare Stellen und Eingriffe durch Herausgeber*innen usw. in der Kodierung darzustellen. Ebenso ist es möglich, umfangreiche Metadaten zur digitalen Edition selbst sowie zu den analogen und digitalisierten Quellen zu integrieren. MEI erlaubt es, all diese Informationen so in ein einzelnes XML-Dokument zu integrieren, dass es sämtliche Daten zur Edition (sozusagen über sich selbst) enthalten kann. Mit diesen semantisch kodierten Daten lässt sich somit alles abbilden, was eine Edition unabhängig von ihrem Medium ausmacht: Quellenbeschreibung, Werkgenese, Herausgeber*inneneingriffe, Editionsrichtlinien usw.

5 Vgl. Roland S. Kamzelak, „Empfehlungen zum Umgang mit Editionen im digitalen Zeitalter”, in: editio 26 (2012), S. 202-209 (DOI 99.9999/edit.2011.018).

6 Vgl. Johannes Kepper, Musikedition im Zeichen neuer Medien (= Schriften des Instituts für Dokumentologie und Editorik 5), Norderstett 2011, S. 226-232.

7 <https://music-encoding.org/update/2018/11/01/mei40.html> (25.06.2020). 
Das Kodierungsziel, das die vermutlich höchsten Ansprüche an Datenstruktur und -qualität stellt, ist das Ermöglichen computergestützter Analyse. Damit ein Programm möglichst effizient arbeiten kann und selbst nicht unnötig komplex gestaltet werden muss, ist es wesentlich, dass die zu verarbeitenden Daten sehr sorgfältig kodiert sind. So genügt es nicht, sich an Schemata zu halten (was in MEI obligatorisch ist), sondern es sollten z.B. auch identische Sachverhalte und Parallelstellen jeweils identisch kodiert werden, um teils aufwendige Berechnungen zu vermeiden. Dazu muss direkt angemerkt werden, dass es in der Regel um grundlegende, häufig quantitative, statistische Auswertungen geht und um das Durchsuchen größerer Korpora, die für traditionelle Arbeit zu umfangreich sind. ${ }^{8}$ Diese Analysen verfolgen zunächst unterstützende und vorbereitende Arbeit, um tiefergehende Forschung zu ermöglichen und im besten Fall sogar neue Fragestellungen und Methoden aufzuwerfen. Eine tiefergehende Analyse, wie sie in der Musikwissenschaft lange Tradition hat, ist noch eine große Herausforderung, an der jedoch intensiv gearbeitet wird. ${ }^{9}$ Die Stärke und der große Nutzen von Computern liegen in ihrer enormen Geschwindigkeit. Notwendige Bedingung hierfür ist die Maschinenlesbarkeit der Daten und die semantische Erfassung ihrer Informationen. In MEI ist dies für gängige musikalische Parameter gegeben, nicht jedoch für Neue Musik mit ihren zuvor beschriebenen Eigenschaften.

Notation abseits von Konventionen und Normen stellt für sich genommen bereits eine immense Herausforderung dar; Paratexte bringen noch weitere eigene Anforderungen an eine sinnvolle Kodierung. Ein Vorwort oder verbalisierte Anweisungen an Interpreten können ohne Probleme in TEI kodiert und mit MEI verknüpft oder in dieses integriert werden. Zusammenhänge und Querverbindungen lassen sich über Referenzen herstellen und Editionen erschöpfend ausführen. Ist jedoch eine computergestützte Analyse ein gestecktes Ziel, genügt es nicht, in MEI die musikalischen Parameter im Text als Freitext ohne semantische Information auszuzeichnen. Zudem ist hier ein neuartiger Quellentyp erkennbar, den man als „technisches Dokument" bezeichnen kann. Es kann aus Text, Grafiken und strukturierten Daten wie Tabellen bestehen. Der in Abbildung 1 wiedergegebene Plan zu Zwischenzonen zeigt dies deutlich: Zu sehen ist im linken Teil eine Tabelle, die den zeitlichen Ablauf des Stücks in den verschiedenen medialen Ebenen (Video, Licht, Tanz, Sprecher/Sänger, Musik, Sampling Digital Audio Tape) sehr übersichtlich darstellt. Im rechten Drittel findet sich quasi ein anderer Quellentypus, nämlich ein grafischer Plan des Bühnenaufbaus für die Uraufführung, der sogar die Namen der

8 Als weit verbreitetes Werkzeuge seien hier die Python-Bibliothek music21 <http://web.mit.edu/music21/> (25.06.2020) oder das 2018 abgeschlossene Dissertationsprojekt von Dr. David Hofmann, music processing suite <https://www.musicprocessing.net/> (25.06.2020), erwähnt.

9 Als stellvertretende Beispiele seien im Bereich von MEI bzw. der Analyse symbolisch kodierter Musik das ÖAWProjekt Digitale Musikanalyse mit den Techniken der Music Encoding Initiative (MEI) am Beispiel der Kompositionsstudien Anton Bruckners <https://www.oeaw.ac.at/acdh/musikwissenschaft/forschung/digital-musicology/ digitale-musikanalyse-mit-mei/> (25.06.2020) genannt und im Bereich der audiobasierten Forschung (Music Information Retrieval) das DFG-Projekt Computer-Assisted Analysis of Harmonic Structures (CAS) <https://www. audiolabs-erlangen.de/fau/professor/mueller/projects> (25.06.2020). 
Interpret*innen enthält. Um diese technischen Dokumente musikalisch-semantisch kodieren zu können, müsste MEI angepasst werden.

Für diesen Zweck werden XML-Elemente, die die neuen Geräte der Live-Elektronik beschreiben können, benötigt. Hier müssen sehr viel mehr Details integriert werden, als das bei konventioneller Musik der Fall ist: Wo sich traditionelle Instrumente wie Klaviere jeweils herstellerübergreifend gleich oder zumindest extrem ähnlich bedienen lassen, ist es nicht notwendig, „Bedienungsanleitungen“ für eine Edition oder Analyse bereitzustellen. Bei Geräten der LiveElektronik jedoch müssen solche Informationen detailliert erfasst werden. So kann die genaue Modellbezeichnung von Bedeutung sein, da sich selbst vermeintlich ähnliche Geräte doch sehr unterschiedlich verhalten können. Darüber hinaus verbessert eine Kategorisierung der Geräte die Übersichtlichkeit; so können sie entweder Klänge selbst erzeugen oder existierende Klänge manipulieren. Die Arten der Klangsynthese (z.B. additiv, subtraktiv, Frequenz- oder Amplitudenmodulation) oder der Manipulation (z.B. Hall, Verzerrung, Kompression) sind ebenfalls von großer Relevanz. So vielseitig wie die Geräte sein können, betrifft das auch deren Steuerung. Es gibt Drehregler, Schieberegler, Taster, Schalter uvm., die unterschiedliche Zahlenbereiche und -skalen (linear, logarithmisch) abdecken. Es liegt hier also ein ungewohntes Niveau an Abstraktion vor: ein sehr geringes. Das Resultat ist ein erheblich vergrößerter Umfang von Kodierungen, da bei Live-Elektronik je nach Zielsetzung einer Kodierung/Edition besonders viele Informationen sehr detailliert erfasst werden müssen und es diesbezüglich quasi keine Konventionen gibt. Informationen sind also kaum durch historische oder stilistische Kontexte gegeben, sondern teils ausschließlich durch Paratexte, um z. B. eine computergestützte Analyse zu ermöglichen.

Komponist*innen experimentieren mit der räumlichen Anordnung von Musiker*innen, Technik (Mikrofone, Lautsprecher) oder sogar dem Publikum. Dafür zeichnen sie Bühnenpläne. Somit muss auch der Parameter Raum in Bezug auf Live-Elektronik wie in Krebs' Komposition Zwischenzonen eingeschlossen werden. Diese Beispiele für neue oder erweiterte Parameter und Quellentypen sind bei weitem nicht erschöpfend (und nicht zwingend exklusiv für Neue Musik und Live-Elektronik); so ist es ein Ziel der Dissertation, diese ebenfalls zu sammeln, zu kategorisieren und in einem weiteren Schritt in den zu entwickelnden Lösungsansatz zur Kodierung Neuer Musik zu integrieren. Dies soll idealerweise auch in anderen Gattungen Anwendungen finden oder zumindest Denkanstöße liefern können.

Mit dem Fortschreiten der Technik wird Musik immer häufiger multimedial, so auch das Beispiel von Joachim Krebs. Musiker*innen, Tänzer*innen, Sprecher*innen und Sänger*innen werden begleitet von Live-Elektronik, Lichttechnik und Videosequenzen. Die Parallelen zur Oper sind klar erkennbar. Neben Partitur, Libretto und Regieanweisungen treten hier weitere Ebenen hinzu. Diese zu kodieren, ist nicht Teil des Dissertationsvorhabens, aufgrund einiger Gemeinsamkeiten sind dessen Ergebnisse aber potenziell zumindest in Teilen hier anwendbar. 
In Anbetracht der enormen Vielfalt neuer elektrischer und elektronischer Instrumente und Geräte, die ständig neu entwickelt werden, sowie der Individualität und Verweigerung von Konventionen oder eigener Neuentwicklungen von Notationsverfahren vieler Komponist*innen würde MEI bei den sich dadurch ergebenden Änderungen zu überfrachtet werden, um noch effizient verwendet werden zu können. Bei Paratexten, die diese dokumentieren, existieren keinerlei Konventionen. Jede ${ }^{\star}$ K Komponist*in entscheidet völlig frei, welche Informationen in welcher Form mitgeliefert werden. Paratexte sind somit auch nicht getrennt vom Notentext zu betrachten, sondern immer in ihrer Beziehung zu ihm. Eine Unzahl an Elementen und Attributen müsste ständig angepasst oder ergänzt werden, um mit der Entwicklung der Musik schritthalten zu können. Dies ist mit den gegebenen Ressourcen weder möglich noch wäre es wünschenswert. Interoperabilität, also die Möglichkeit, Dateien verschiedener Herkunft zu kombinieren und konsistente Informationen zu erhalten, wäre kaum leistbar, denn mit dem Umfang von MEI wächst auch die Zahl an Möglichkeiten, mit musikalischen Inhalten umzugehen. Schon bei "traditionellen" Editionsprojekten ist ein Austausch von MEI-Daten derart komplex, dass eigens MEI Basic als Untermenge von MEI und gemeinsame Basis entwickelt wird, vergleichbar mit dem größten gemeinsamen Teiler in der Mathematik. MEI leichtfertig zu ergänzen, wäre für diese Problematik kontraproduktiv.

Das Poster wirft ein Schlaglicht auf Paratexte, die somit eine deutlich erweiterte Bedeutung erhalten und vermehrt technische Informationen in Formen enthalten, die weder mit MEI noch anderen verfügbaren Formaten adäquat kodierbar sind. Um Kodierungen und digitale Editionen Neuer Musik vollumfänglich zu ermöglichen, ist es notwendig, neben der eigentlichen Notation auch diese Quellentypen zu berücksichtigen und sie in den Lösungsansatz, der in der Dissertation entwickelt werden soll, zu integrieren. Eine schlichte Erweiterung von MEI und/ oder TEI wäre dabei zu kurz gedacht.

Zitation: Daniel Fütterer, „Herausforderungen bei der Kodierung von Paratext am Beispiel Neuer Musik mit LiveElektronik", in: Brückenschläge zwischen Musikwissenschaft und Informatik. Theoretische und praktische Aspekte der Kooperation, in Verbindung mit der Fachgruppe Digitale Musikwissenschaft hrsg. von Stefanie Acquavella-Rauch, Andreas Münzmay und Joachim Veit (= Musikwissenschaft: Aktuelle Perspektiven. Bericht über die Jahrestagung der Gesellschaft für Musikforschung 2019 in Paderborn und Detmold, Bd. 3), Detmold, Musikwissenschaftliches Seminar der Universität Paderborn und der Hochschule für Musik Detmold, 2020, S. 141-148, DOI: 10.25366/2020.103 


\section{Abstract}

In traditional scholarly editions of music, peritext only plays a minor role so far. Peritexts can be defined as integral components of a score, for example a foreword, that are not part of the musical text. Especially in New Music, which is often intentionally breaking with implicit performance traditions, peritexts might offer essential information, e. g. on the arrangement of instruments and personal on the stage, about the used effects and hardware, and on verbal instructions to the interpreters. Encoding this information to be fully accessible for a scholarly digital music edition, is an important challenge. The poster is explaining this issue using an example by Joachim Krebs.

\section{Kurzvita}

Daniel Fütterer, geb. 1989 in Karlsruhe, studierte an der Hochschule für Musik Karlsruhe von 2010-14 Musikinformatik B.A. und von 2014-17 Musikwissenschaft M.A. Seit 2018 arbeitet er an seiner Promotion über die Kodierung Neuer Musik mit dem Ziel, Live-Elektronik, die meist graphisch notiert ist, besser erfassbar zu machen. Seit 2019 arbeitet er als wissenschaftlicher Mitarbeiter am Institut für Musikinformatik und Musikwissenschaft an der HfM Karlsruhe. 


\section{Herausforderungen bei der Kodierung von Paratext am Beispiel Neuer Musik mit Live- Elektronik}

\section{Abstract}

Paratexte sind wichtige Quellen, die zum Teil wesentliche Informationen enthalten. Aufgrund ihrer vielfältigen Erscheinungsformen stellen sie für die Kodierung eine immense Herausforderung dar, die sich bei Musik mit Live-Elektronik noch einmal zugespitzt zeigt.

Das Poster beleuchtet diese Thematik im Rahmen meines laufenden Dissertationsprojektes.

\section{Kontakt}

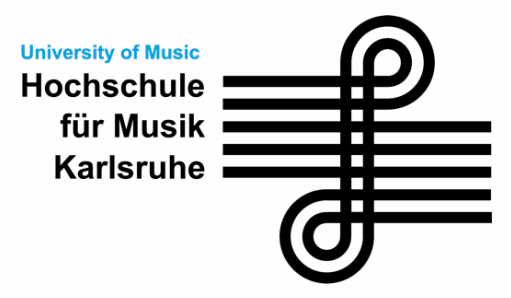

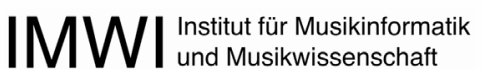

\section{Daniel Fütterer, M.A. \\ fuetterer@hfm-karlsruhe.de}

Betreuer:

Prof. Dr. Thomas Troge

Prof. Dr. Stefanie Steiner-Grage

Prof. Dr. Joachim Veit

Unterstützer:

Prof. Dr. Christoph Seibert

Sabine Schäfer

Badische Landesbibliothek Karlsruhe

\section{Neue Parameter}

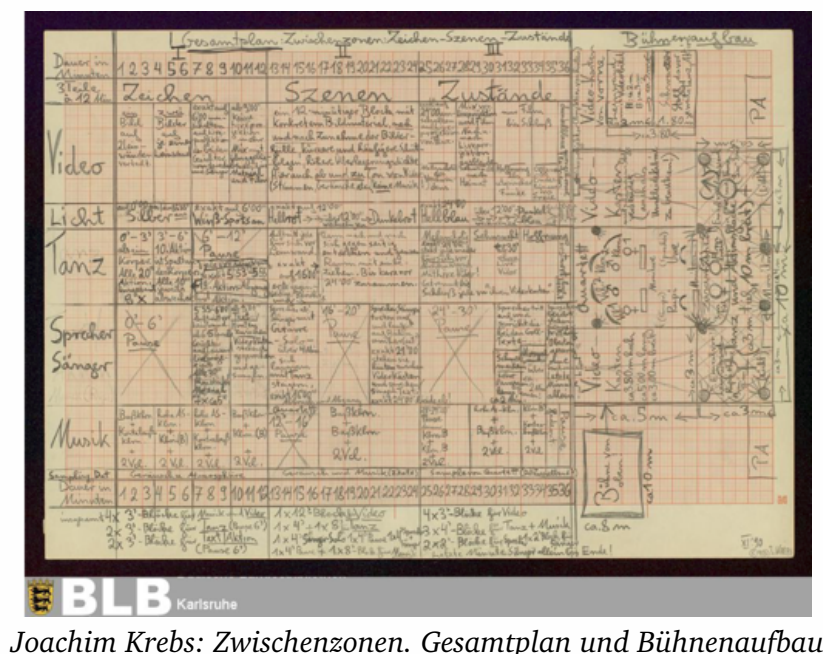

FRAGESTELLUNGEN:

- Warum spielen Paratexte ausgerechnet bei Neuer Musik eine große Rolle?

- Welche Auswirkungen auf die Kodierung hat Live-Elektronik?

\section{ERKLÄRUNGSANSATZ:}

Der Bedeutungsverlust des impliziten Wissens Für Musik vom 17. bis 19. Jahrhundert ist die Notation und Umsetzung bis zu einem gewissen Grad »einheitlich«. Im 20. Jahrhundert begannen Komponistinnen und Komponisten, mit diesen Konventionen zu brechen: Ideen ließen sich nicht mit dem bewährten System notieren, es schränkte ein und wurde abgelegt; der Bruch mit der Tradition war oft forciert.

Was zuvor durch Konventionen bekannt war und nicht Teil der Notenausgabe sein musste, fehlte nun. Diese Informationen waren nicht Bestandteil des Notentextes, sondern eines umfangreichen Vorworts der Partitur, beigelegten Skizzen oder Plänen.

Live-Elektronik Der Einsatz von Live-Elektronik verkompliziert die Problematik deutlich. Zu Unterschiedlich sind die Geräte und zu vielfältig ihre Bedienung, als dass sich für sie überhaupt eine Notations-Konvention hätte entwickeln können. Die Belegung eines Mischpultes kann ebenso relevant werden wie die Anordnung verwendeter Lautsprecher.

\section{SCHLUSSFOLGERUNG:}

Um ein Werk mit all seinen (neuen) Parametern verstehen zu können, genügt es nicht, sich ausschließlich mit dem Notentext zu befassen - auch Paratexte müssen miteinbezogen werden.

\section{Kodierung}

Den Notentext zu kodieren, ist gegenwärtig die beste Methode, um eine möglichst vielfältige Nachnutzung zu ermöglichen. Eine Kodierung kann dabei mehrere Ziele verfolgen, die jeweils eigene Anforderungen an diese stellen:

- Archivierung, Sicherung und Publikation der Quelle

- Genuin digitale Edition eines Werks

- Datengrundlage für eine computergestütze Analyse

Für das erste Ziel, eine Quelle in ihrem Zustand zu archivieren und zugänglich zu machen, genügt oft ein herkömmliches Digitalisat. Ist das Ziel eine Edition oder Analyse, ist jedoch eine tiefe Kodierung notwendig, die auch Semantik beschreiben kann.

MEI (Music Encoding Initiative), der etablierte Standard, wurde jedoch nicht für Neue Musik und ihre Notation konzipiert. MEI ermöglicht es, angepasst zu werden - unbeantwortet ist aber die Frage, was die beste Methode dafür ist. Dabei treten folgende Probleme auf:

- Umfang der Anpassungen

- Hohe Individualität der Komponisten/Werke

- Erhaltung von Interoperabilität

\section{Bildnachweis}

Badische Landesbibliothek Karlsruhe: Signatur K 3353 A 43 



\section{Brückenschläge zwischen Musikwissenschaft und Informatik}

Theoretische und praktische Aspekte der Kooperation

Herausgegeben von Stefanie Acquavella-Rauch,

Andreas Münzmay und Joachim Veit 
Brückenschläge zwischen Musikwissenschaft und Informatik 


\section{Musikwissenschaft: Aktuelle Perspektiven}

Bericht über die Jahrestagung der Gesellschaft für Musikforschung 2019 in Paderborn und Detmold

Herausgegeben von Rebecca Grotjahn und Nina Jaeschke

Band 3 


\section{Brückenschläge zwischen Musikwissenschaft und Informatik}

Theoretische und praktische Aspekte der Kooperation

Beiträge der Symposien zur Digitalen Musikwissenschaft

Osnabrück 2018 und Paderborn 2019

im Rahmen der Jahrestagungen der Gesellschaft für Musikforschung

In Verbindung mit der Fachgruppe Digitale Musikwissenschaft herausgegeben von

Stefanie Acquavella-Rauch, Andreas Münzmay und Joachim Veit

Detmold: Musikwissenschaftliches Seminar der Universität Paderborn und der Hochschule für Musik Detmold 2020 
DOI: $10.25366 / 2020.87$

Online-Version verfügbar unter der Lizenz: Urheberrecht 1.0, $<$ https://rightsstatements.org/page/InC/1.0/?language =de >

Bibliografische Information der Deutschen Nationalbibliothek

Die Deutsche Nationalbibliothek verzeichnet diese Publikation in der Deutschen Nationalbibliografie; detaillierte bibliografische Daten sind im Internet über http://dnb.d-nb.de abrufbar.

\section{Impressum}

Redaktion: Stefanie Acquavella-Rauch, Andreas Münzmay und Joachim Veit Satz: Nina Jaeschke und Joachim Veit

(C) Musikwissenschaftliches Seminar der Universität Paderborn und der Hochschule für Musik Detmold 2020 


\section{INHALT}

Rebecca Grotjahn, Nina Jaeschke

Vorwort zu Band 1-3

IX

Stefanie Acquavella-Rauch, Andreas Münzmay, Joachim Veit

Brückenschläge zwischen Musikwissenschaft und Informatik - Vorbemerkung

$\mathrm{XI}$

\section{KOLLABORATIONEN - KO-LABORATORIEN}

\section{Reinhard Keil}

Der Computer als Denkzeug für hermeneutische Arbeit

\section{Ulrich Konrad}

Philologie und Digitalität. Perspektiven für die Musikwissenschaft im Kontext fächerübergreifender Institutionen

\section{Gudrun Oevel}

Infrastruktureinrichtungen in Forschungsprojekten - Spagat oder Chance?

\section{Dennis Ried}

Erhebung, Transformation und Präsentation digitaler Forschungsdaten

Anna Neovesky, Frederic von Vlahovits

IncipitSearch - Leitfaden zur Zusammenarbeit

Elisabeth Treydte

Clara Schumann \#digital. 40 Jahre Archiv Frau und Musik und der Start in die Digitalisierung

\section{TEXT/DATEN/PROZESSE}

\section{Christine Siegert}

Komponisten-Gesamtausgaben im digitalen Zeitalter: Perspektiven

und Reflexionen am Beispiel Ludwig van Beethovens

Markus Neuwirth, Johannes Hentschel, Martin Rohrmeier

Perspectives of Musical Corpus Studies: The Annotated Mozart Sonatas

Agnes Amminger, Franz Kelnreiter

Leopold Mozarts „Gründliche Violinschule". Zur Textcodierung und -präsentation einer digitalen Edition

\section{Oleksii Sapov}

Algorithmische Automatisierung komplexer Notationsregeln in MEI-XML am Beispiel von Versetzungszeichen 


\section{Susanne Cox, Richard Sänger}

Digitale Fassungsvergleiche am Beispiel von Beethovens Eigenbearbeitungen

Agnes Seipelt

Digitale Edition und Harmonische Analyse mit MEI von Anton Bruckners

Studienbuch

Stefanie Acquavella-Rauch

Musikalische Schaffensprozesse 2.0 - Inkorporation audiovisueller Medien

der populären Musik in Methoden der digitalen Edition

\section{DIGITAL(ISIERT)E MATERIALITÄTEN}

\section{Miriam Akkermann}

(Musik)Instrument (im) Computer

\section{Daniel Fütterer}

Herausforderungen bei der Kodierung von Paratext am Beispiel Neuer Musik mit Live-Elektronik

\section{Matthias Pasdzierny}

How much is the glitch? Das digitale Paradigma als Herausforderung

und Chance für die historische Musikwissenschaft

\section{Shintaro Miyazaki}

Musik für Maschinen?! - Wo sich die Wissenschaft der Medien, des Computers und der Musik treffen und wie sie zusammenarbeiten könnten

\section{MUSIKGESCHICHTE(N) IM NETZ}

\section{Matthias Tischer}

Musikgeschichte der DDR: Ein Pilotprojekt zur digitalen Musikvermittlung

Annette van Dyck-Hemming, Jan Eberhardt, Melanie Wald-Fuhrmann

Ansätze zur Analyse historischer Netzwerke mit Neo4j® - Aus der Projekt-Werkstatt der Datenbank zur Fachgeschichte der Musikwissenschaft

Axel Beer, Martin Bierwisch, Kristina Krämer

Das MMM2 - Ein regionalgeschichtliches Onlinelexikon der Arbeitsgemeinschaft für mittelrheinische Musikgeschichte

\section{Matej Santi}

Was erzählt Fritz Kreislers Geige?

\section{Elias Berner}

Alle Menschen werden Brüder?! Ein historisches Dokument aus dem

Nationalsozialismus in den sozialen Medien 
Gabriele Buschmeier in memoriam 